\title{
Recurrence of carcinoma of the lower lip treated by interferon and irradiation
}

\author{
Boris Jančar \\ Department of Radiation Oncology, Institute of Oncology, Ljubljana, Slovenia
}

A seventy-year-old patient was referred to our hospital for a tumour on the lower lip, which had been growing for the last two years. On the first clinical examination, the tumour, measuring $3.5 \mathrm{~cm}$ in diameter, was visible to the naked eye. Histological examination of the biopsy sample confirmed verrucous carcinoma.

The patient had been treated for miocardiopathy and emphysema for several years. Though at rest, the patient was breathing heavily and suffered from oedemas and small ulcers on both shanks. It was thus evident that the patient was not eligible for surgery under general anaesthesia, but rather for irradiation therapy.

The irradiation was performed by an orthovolt machine (PANTAC) with a total dose of 40 Gy (10 x 4 Gy). The tumour regressed completely. Six months after the completed therapy, a minor lesion, which would not heal up, developed on the tumour site. Several subsequent fine-needle aspiration biopsy (FNAB) examinations did not detect any malignant cells in the lesion. The patient complained that the lesion was painful. Two years after the completed radio-

Received 5 February 2007

Accepted 15 February 2007

Correspondence to: Prim. Boris Jančar, MD, MSc, Department of Radiation Oncology, Institute of Oncology, Zaloška 2, Ljubljana, Slovenia; Phone; + 38615879 295; Fax: + 38615879 295; E-mail: bojancar@onko-i.si

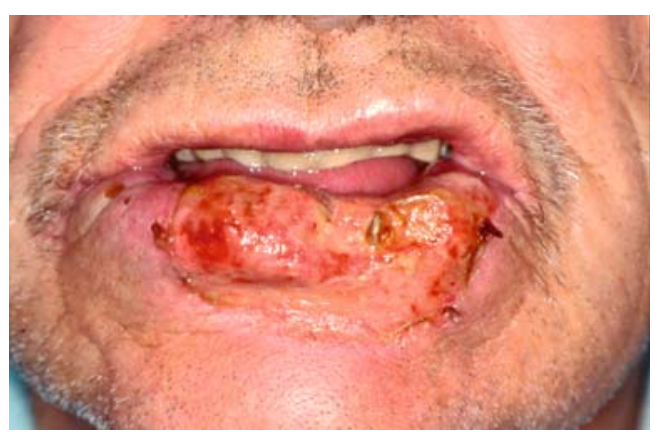

Figure 1. Recurrence of the tumour after radiotherapy.

therapy, a tumour with a diameter of $1 \mathrm{~cm}$ appeared on the lower lip on the same site as the primary which completely regressed after the therapy, but obviously recurred two years later. The patient, believing that the tumour would fade away by itself, refused surgical treatment under local anaesthesia.

In the following two years, the tumour was growing further and four years after the completed radiotherapy measured $3 \mathrm{x}$ $2 \mathrm{~cm}$ (Figure 1). FNAB examination confirmed the recurrence of the squamous cell carcinoma. Due to poor physical condition of the patient, surgical treatment was most unlikely. The patient was therefore treated with the interferon injected directly into tumour. The tumour partially regressed after 5 injections and the therapy with interferon was then combined with irradiation. The patient was receiving 3 Mil IE per application and was submitted to hyperfractiona- 
ted radiotherapy (38 fractions, $2 \times$ /day, 1.5 Gy/fraction). In two months' time, the tumour regressed. Nine months later the cosmetic effect was quite good (Figure 2). At the last follow-up control three years after the second therapy, the lower lip was NED.

As the patient's poor physical condition did not improve and the transfer from home to the hospital and back were getting more and more physically exhausting for him, he was not invited to follow-up controls any more. He died of myocardial infarction 4.5 years after the last therapy for the tumour, with NED on the lower lip.

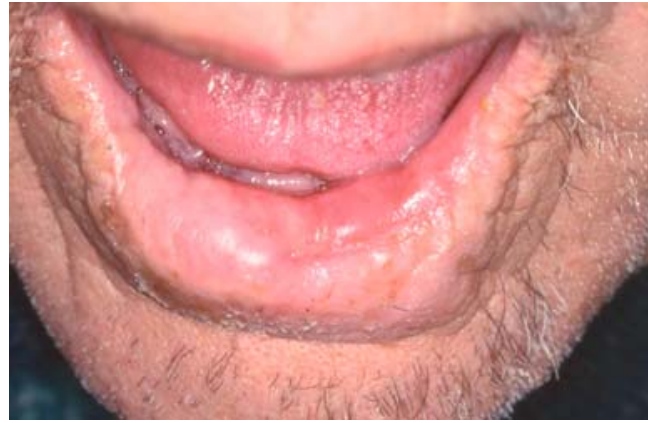

Figure 2. Nine months after combined treatment with irradiation and interferon. 